\section{Dueling interferons}

Most individuals infected with mycobacteria can clear or contain these intracellular parasites, but others may develop disseminated infections. In Science, Modlin and colleagues show that the control of mycobacterial infections is dictated by the type of interferon (IFN) produced. Patients with self-healing tuberculoid leprosy, caused by Mycobacterium leprae, express more IFN- $\gamma$, leading to the upregulation of vitamin D-dependent antimicrobial peptides, such as cathelicidin and $\beta$-defensin 2. In contrast, patients with disseminated lepromatous leprosy express more IFN- $\beta$ and show increased expression of IFN- $\beta$ dependent genes, including more IL-10. Blocking IL-10 reduces the bacterial load in infected human monocytes. Analysis of published tuberculosis gene expression profiles reveals a similar dichotomy of IFN- $\gamma$ - and IFN- $\beta$-mediated responses in resolving as opposed to chronic forms of the disease. These findings could be used to combat chronic mycobacterial infection by modulating IFN- $\beta$ and IL- 10 . $L A D$ Science 339, 1448-1453 (2013)

\section{HIV turns off the TCR}

HIV-1 env (envelope) encodes two non-covalently associated proteins, gp41 and gp120, that are involved in virus entry into target cells and in immune modulation. In Blood, Shai and colleagues reveal a further mechanism by which gp41 can inhibit $\mathrm{CD} 4^{+} \mathrm{T}$ cells directly. The authors identify a conserved motif within the gp41 loop region that, when expressed as a peptide, can inhibit cognate T cell activation in vitro as well as in an in vivo mouse model of experimental autoimmune encephalitis. Proteinmembrane interactions are typically dependent on hydrophobic residues, and accordingly tryptophan repeats are often used to facilitate this process. Interestingly, the inhibitory action of the gp41 motif also depends on a tryptophan repeat. Mutagenesis data suggest that these tryptophan repeats allow gp41's interaction with the T cell receptor (TCR) alpha chain within the cell membrane and thereby disrupt proper assembly and signaling of the TCR-CD3 complex.

Blood 121, 2244-2252 (2013)

\section{RLR phosphorylation}

RIG-I and Mda5 are key cytosolic sensors for the detection of RNA viruses and the production of interferon. In Immunity, Wies et al. show that Mda5 activation is regulated by a dynamic balance between phosphorylation and dephosphorylation of its $\mathrm{N}$-terminal CARD domains. Phosphorylation of Mda5 at Ser88 residue under normal conditions suppresses interaction of Mda5 with MAVS. The phosphatases PP $1 \alpha$ and PP $1 \gamma$ specifically dephosphorylate this residue, as well as Ser8 in RIG-I, to induce MAVS-CARD interactions, downstream signaling and interferon production. Virus infection triggers the recruitment of $P P 1 \alpha$ and PP $1 \gamma$ to Mda5 and RIG-I. Overexpression or depletion of the two phosphatases, which have additive effects, modulates the efficiency of viral replication in infected cells. These results add phosphorylation to the list of post-translational modifications that control the activity of RIG-I-like receptors.

Immunity 38, 437-449 (2013)

\section{Trib1, M2s and the adipose tissue}

Mutations in TRIB1 have been implicated in human metabolic disorders. In Nature, Akira and colleagues show that Trib1, an adaptor protein that plays a part in protein degradation by interacting with the E3 ubiquitin ligase COP1, is critical for the differentiation of tissueresident M2 anti-inflammatory macrophages and eosinophils, but not M1 pro-inflammatory macrophages and neutrophils, in mice. Trib1 controls myeloid cell differentiation by altering the expression of the transcription factor $\mathrm{C} / \mathrm{EBP} \alpha$ in a COP1-dependent manner. Trib1 ${ }^{-/}$ mice have reduced abdominal adipose tissue when fed a normal diet and develop glucose intolerance and insulin resistance, without an increase in body weight, on a high-fat diet. Reconstitution with wild-type M2 macrophages rescues the lipodystrophy of Trib1 $1^{-/-}$mice, suggesting that tissue-resident M2 macrophages control adipose tissue homeostasis and possibly progression to metabolic disorders. IV Nature (20 March 2012) doi:10.1038/nature11930

\section{Putting a block on Foxp3}

The function of regulatory $T\left(T_{\text {reg }}\right)$ cells is known to be impaired in rheumatoid arthritis (RA). In Nature Medicine, Zhang and colleagues delineate the molecular mechanism underlying this impairment. The transcription factor Foxp3 is fundamental to $T_{\text {reg }}$ cells' suppressive abilites and for defining their cellular identity; however, certain inflammatory environments can perturb its activity. The authors show that synovial fluid from patients with $R A$ reduced $T_{\text {reg }}$ cell activity and anergy and that the inflammatory cytokine TNF was uniquely responsible for this. In patients with RA and in TNF-treated $\mathrm{T}_{\text {reg }}$ cells, $\mathrm{T}_{\text {reg }}$ cell frequency and their Foxp3 expression appear normal, but phosphorylation of Foxp3 is reduced. Impairment of Foxp3 activity reduced its ability to repress $T_{\text {reg }}$ cells' IL-2 expression. Knockdown and immunoprecipitation show that expression of the phosphatase PP1 is increased by TNF; PP1 associates with Foxp3 and thereby reduces its phosphorylation. TNF blockade in patients with RA reduces PP1, restores Foxp3 phosphorylation and rescues $T_{\text {reg }}$ cell suppressive function. These findings suggest another level at which TNF-blockade may ameliorate autoinflammatory disease.

Nat. Med. 19, 322-328 (2013)

\section{Pu. 1 in HSCs}

Relative abundance of PU.1, a transcription factor essential during hematopoiesis, controls lineage fate decisions. In Molecular Cell, Staber et al. examine PU.1 activity in mouse hematopoietic stem cells (HSCs). PU.1 regulates its own expression by binding to the promoter and an upstream regulatory element (URE) at $-14 \mathrm{~kb}$ relative to the transcriptional start site. Mutation of the URE Pu.1 site decreases expression of PU.1 in HSCs but not macrophages. Mice harboring such hypomorphic mutations have HSCs that show reduced longterm repopulation potential and exhibit hematopoietic failure after repeated stressing. PU.1 acts by inhibiting genes that promote cell proliferation and activates those encoding cell-cycle inhibitors. Thus PU.1 is required to maintain HSC quiescence and prevent exhaustion of the HSC pool.

$L A D$ 